\title{
FILTROS CÔNICOS COM CAPACIDADE MÁXIMA
}

Richard Mariano de Souza Silva, Fernando Pereira de Souza, Eugenia Brunilda Opazo Uribe.

Universidade Federal de Mato Grosso do Sul - UFMS, Curso de Licenciatura em Matemática, Três Lagoas, MS. E-mail: richard.mariano.mat@gmail.com.

Agência de fomento: Programa de Educação Tutorial

\section{RESUMO}

O presente artigo é resultado de um trabalho de pesquisa de um aluno do Curso de Matemática no âmbito do Programa de Educação Tutorial, desenvolvido com o objetivo de explorar aplicações matemáticas relacionadas com otimização de grandezas no parâmetro industrial. O trabalho foi desenvolvido através de pesquisa bibliográfica e estudo teórico relacionado, apresentações de seminários e resolução de exercícios. Será trabalhado a aplicação da matemática em função da projeção de filtros cônicos com capacidade máxima, levando em consideração o caso com descarte e sem descarte.

Palavras-chave: Filtros, Aplicações, Programa de Educação Tutorial.

\section{CONICAL FILTERS WITH MAXIMUM CAPACITY}

\section{ABSTRACT}

This article is the result of a research work of a student of the Mathematics Course within the scope of the Tutorial Education Program, developed with the objective of exploring mathematical applications related to optimization of quantities in the industrial parameter. The work was developed through bibliographical research and theoretical study related, including seminars, exercises. The application of mathematics will be studied in function of the projection of conical filters with maximum capacity, taking and considering the case with discard and without discard.

Keywords: Conical Filters, Applications, Tutorial Education Program. 


\section{INTRODUÇÃO}

A otimização de variáveis é muito comum no âmbito industrial, pois a exigência para se produzir de forma que o desperdício seja mínimo, é algo muito presente. Por isso, é fácil encontrar aplicações matemáticas nesta área. Graças à matemática é possível fazer um estudo de tal forma, que se conheça qual a melhor maneira de se produzir um objeto, sem que se perca matéria prima, evitando assim o desperdício, e consequentemente perda de matéria prima. O presente trabalho irá mostrar uma aplicação matemática na otimização de filtros cônicos, de forma que eles tenham sua capacidade máxima. As imagens presentes no trabalho foram feitas pelo autor, utilizando o programa GeoGebra versão 6.0.385.0 e Paint 3D versão 3.1709.5027.0.

\section{METODOLOGIA}

O trabalho foi desenvolvido através de pesquisa bibliográfica e um estudo teórico que incluiu apresentação de seminários e resolução de exercícios.

\section{RESULTADOS}

Iremos começar problematizando o caso com descarte. Consideraremos um círculo com raio fixo $G$, com ângulo $\alpha$ radianos para a construção do filtro cônico, e $(2 \pi-\alpha)$ radianos sendo o ângulo a ser descartado. O objetivo é determinar um padrão de corte tal que extraia deste círculo o setor que produz o filtro cônico com sua capacidade máxima.

Temos que o comprimento do arco do setor circular coincidirá com o perímetro da abertura do filtro, além de o raio $\mathrm{G}$ coincidir com a geratriz do cone, que irá definir o formato do filtro. Denotaremos por $\mathrm{r}$ o raio da base e por $\mathrm{H}$ a altura do cone, de acordo com a figura,
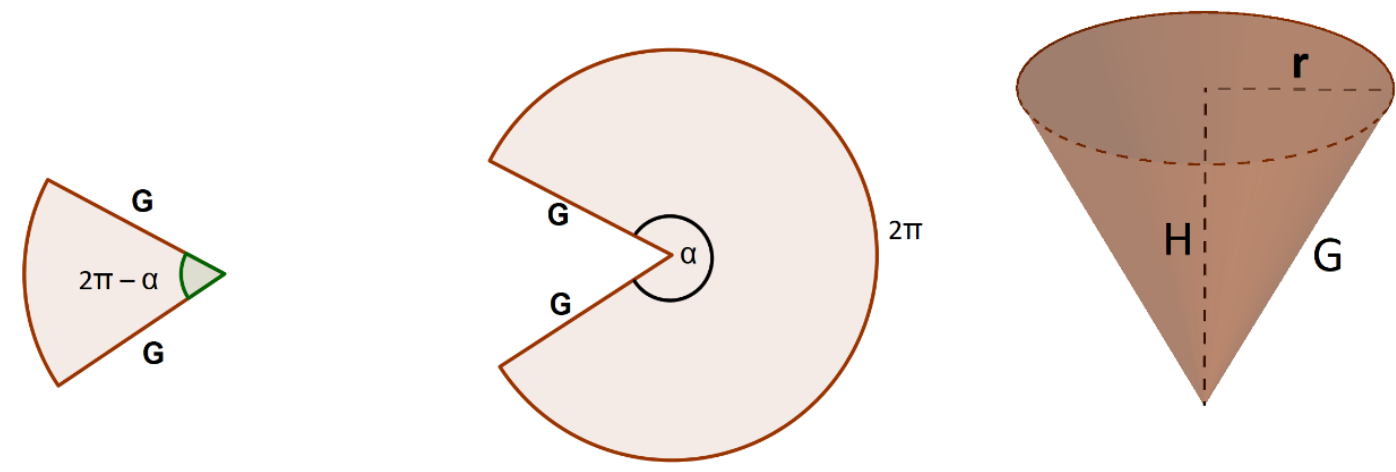

Assim, teremos que $G \times \alpha=2 \pi r$, e portanto $r=\frac{G \times \alpha}{2 \pi}$.

Analisand o o cone que foi construído, podemos afirmar, do Teorema de Pitágoras, que $G^{2}=H^{2}+r^{2}$, logo, $H=\sqrt{G^{2}-r^{2}}$.

Assim, substituindo o valor de $r=\frac{G \times \alpha}{2 \pi}$, na expressão $H=\sqrt{G^{2}-r^{2}}$, temos,

$$
H=\sqrt{G^{2}-\left(\frac{G \times \alpha}{2 \pi}\right)^{2}}=\sqrt{\frac{G^{2}}{4 \pi^{2}}\left(4 \pi^{2}-\alpha^{2}\right)} \text {, então } H=\frac{G}{2 \pi} \times \sqrt{4 \pi^{2}-\alpha^{2}} .
$$

Agora, como a formula do volume de um cone é dada por $V=\frac{1}{3} \pi r^{2} H$, podemos expressar a capacidade do filtro em função do ângulo do setor circular, substituindo o valor de $H$ e de $r$, que já são conhecidos, obtendo,

$$
V(\alpha)=\frac{1}{3} \pi r^{2} \frac{G}{2 \pi} \times \sqrt{4 \pi^{2}-\alpha^{2}}=\frac{1}{3} \pi \frac{G^{2} \times \alpha^{2}}{4 \pi^{2}} \times \frac{G}{2 \pi} \times \sqrt{4 \pi^{2}-\alpha^{2}}=\frac{G^{3}}{24 \pi^{2}} \times \sqrt{4 \pi^{2} \alpha^{4}-\alpha^{6}}
$$


Temos que a capacidade $V$ do filtro se anula quando $\alpha=0$ e $\alpha=2 \pi$, assim iremos restringir o domínio de $V$, sendo ele então $I=] 0,2 \pi$. Como $G^{3} / 24 \pi^{2}$ é uma quantidade fixa, os pontos críticos de $V$ irão coincidir com os pontos críticos de $f(\alpha)=\sqrt{4 \pi^{2} \alpha^{4}-\alpha^{6}}$.

Podemos verificar no gráfico de $f$ que existe um ponto máximo absoluto em $A$, e segundo o software utilizado na plotagem do gráfico (GeoGebra 5.0) as coordenadas deste ponto são: $A(5,1302 ; 95,4746)$.

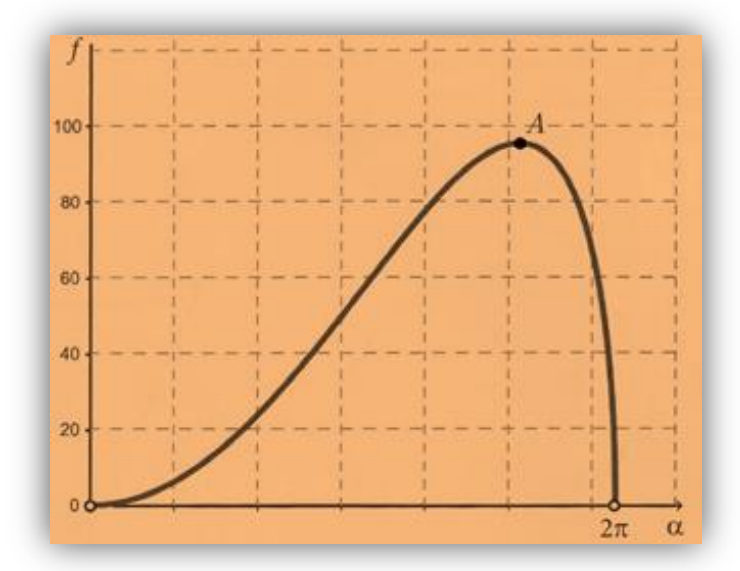

Logo, podemos concluir que o valor aproximado da abcissa maximizada é $\propto=5,1302$ radianos. A expressão final da derivada de $f$ em relação a $\propto$ é:

$$
f^{\prime}(\alpha)=\frac{d f}{d \alpha}=\frac{8 \pi^{2} \alpha^{3}-3 \alpha^{5}}{\sqrt{4 \pi^{2} \alpha^{4}-\alpha^{6}}}
$$

Como $\alpha \neq 0$ e $\alpha \neq 2 \pi$, resta um único ponto crítico para $f$, sendo ele $f^{\prime}(\alpha)=0$, ou seja, quando $8 \pi^{2} \alpha^{3}-3 \alpha^{5}=0$, assim $3 \alpha^{2}=8 \pi^{2}$, quando $\alpha=\frac{2 \pi \sqrt{6}}{3}$ radianos (aproximadamente 5,1302 radianos). filtro é:

Como a abscissa que maximiza $f$ também maximiza $V$, temos que a capacidade máxima do

$$
V\left(\frac{2 \sqrt{6 \pi}}{3}\right)=\frac{G^{3}}{24 \pi^{2}} \times \sqrt{4 \pi^{2} \times\left(\frac{2 \sqrt{6} \pi}{3}\right)^{4}-\left(\frac{2 \sqrt{6} \pi}{3}\right)^{6}}=\left(\frac{2 \sqrt{3} \pi}{27}\right) \times G^{3} \cong 0,403066 G^{3}
$$

Agora sobre o padrão ótimo de corte, temos que ocorrerá o corte do círculo em dois setores, um deles de 5,1302 $\mathrm{rad}\left(294^{\circ}\right)$ que será a parte utilizada, e o outro de 1,1530 rad $\left(66^{\circ}\right)$ que será a parte descartada.

Sobre o perfil (secção meridiana) do filtro ótimo, temos das expressões que: $\frac{r}{G}=\frac{\alpha}{2 \pi}=\frac{\sqrt{6}}{3} \mathrm{e}$ $H=\sqrt{G^{2}-r^{2}}$ delas podemos obter a razão entre o raio e a profundidade da abertura: $\frac{H}{r}=\frac{\sqrt{2}}{2}=\operatorname{tg} 35,3^{\circ}$. O que nos mostra que a figura trabalhada é um triangulo isósceles, com ângulos da base iguais a $\theta=35,5^{\circ}$, como podemos ver na figura abaixo:

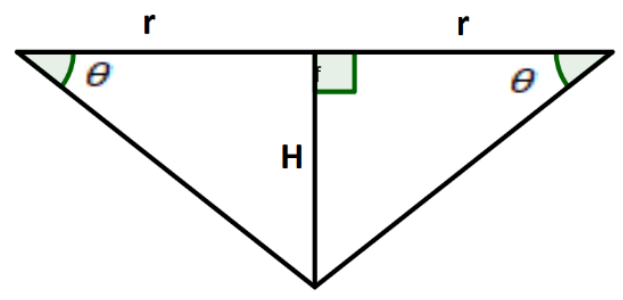


Por fim, vamos analisar a capacidade do filtro que seria obtido com o setor circular descartado. O ângulo do setor descartado é o suplemento do ângulo do setor que foi utilizado para a construção do filtro de capacidade máxima, logo se construíssemos um filtro com ele, sua capacidade seria:

$$
V\left(2 \pi-\frac{2 \sqrt{6} \pi}{3}\right)=V\left(\frac{6 \pi-2 \sqrt{6} \pi}{3}\right)=\left(\frac{5-2 \sqrt{6} \pi}{27}\right) \times \sqrt{6 \sqrt{6}-6} \times \pi \times G^{3} \cong 0,03466 G^{3} .
$$

Agora iremos problematizar o caso sem descarte. Consideremos um círculo de raio fixo $G$, iremos determinar um padrão de corte tal que a soma das capacidades dos dois filtros produzidos seja máxima. Neste caso, tanto o setor de ângulo $\alpha$ radianos quanto o de $(2 \pi-\alpha)$ radianos vão ser utilizados para a construção de filtros.
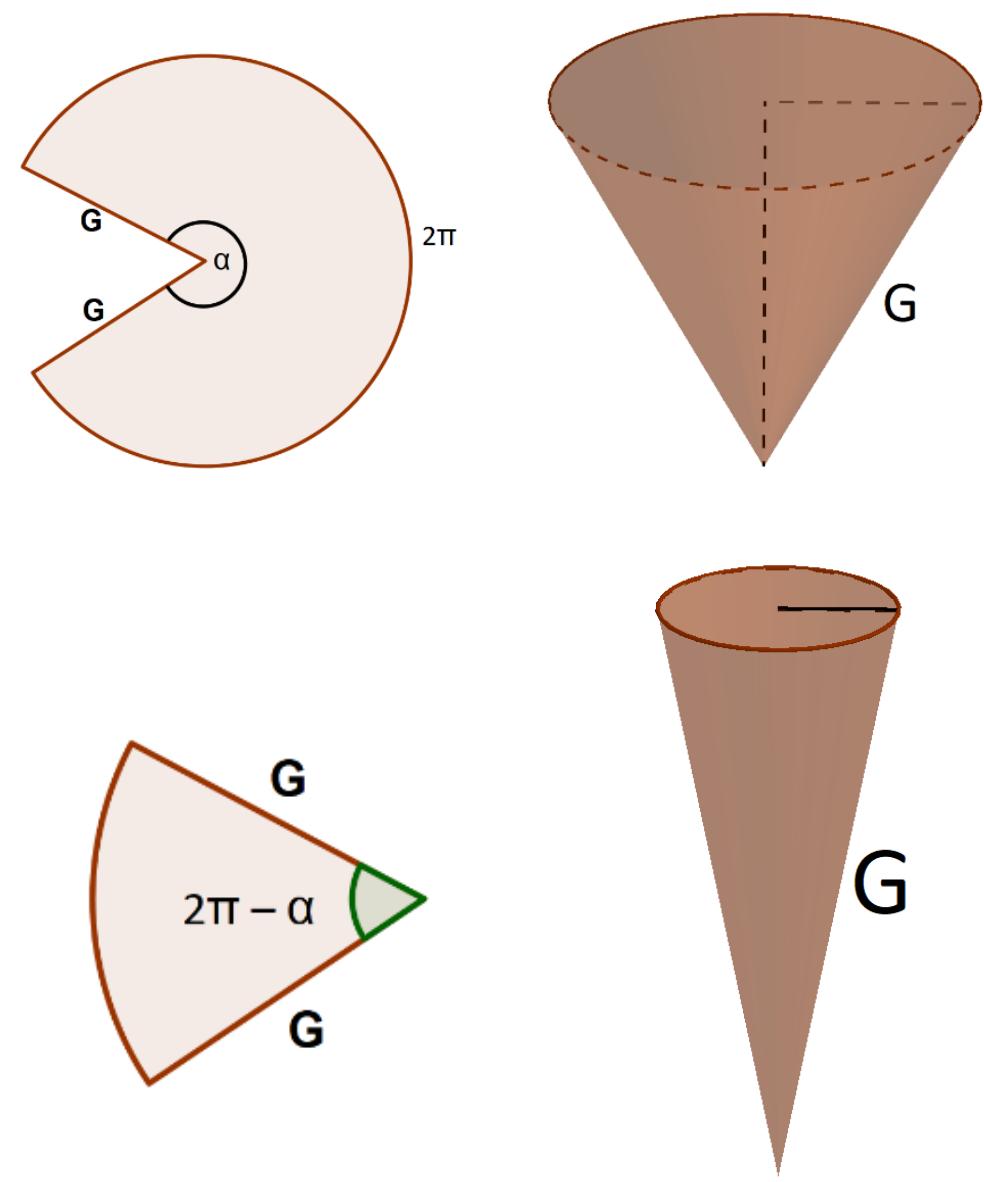

Seja $S$ a função que representa a soma das capacidades dos dois filtros, temos que:

$S(\alpha)=V(2 \pi-\alpha)$,, onde $V$ é $V(\alpha)=\frac{G^{3}}{24 \pi^{2}} \times \sqrt{4 \pi^{2} \alpha^{4}-\alpha^{6}}$, portanto:

$S(\alpha)=\frac{G^{3}}{24 \pi^{2}} \times\left(\sqrt{4 \pi^{2} \alpha^{4}-\alpha^{6}}+\sqrt{4 \pi^{2}(2 \pi-\alpha)^{4}-(2 \pi-\alpha)^{6}}\right)$, iremos restringir os pontos onde $S$ se anula, portanto o domínio de $S$ será $I=] 0,2 \pi[$.

Como $\frac{G^{3}}{24 \pi^{2}}$ é fixo, então os eventuais pontos críticos de $S$ vão coincidir com os da função $\Psi(\alpha)=\sqrt{4 \pi^{2} \alpha^{4}-\alpha^{6}}+\sqrt{4 \pi^{2}(2 \pi-\alpha)^{4}-(2 \pi-\alpha)^{6}}$.

Vemos na imagem abaixo que o gráfico de $\Psi$ é simétrico em relação a reta $\alpha=\pi$ no intervalo $I$. 


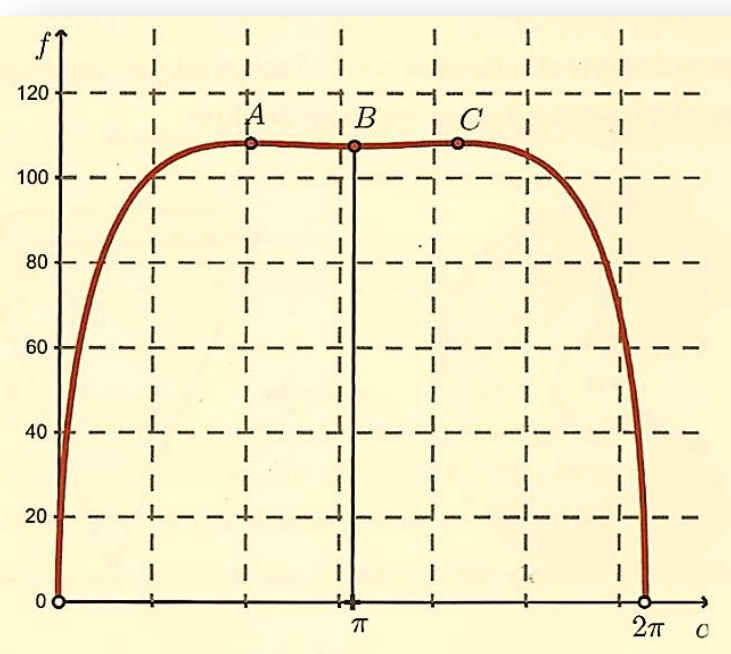

Podemos concluir ao ver o gráfico que existem dois pontos máximos absolutos iguais nos pontos $A$ e $C$, e um mínimo no ponto $B$. Segundo o software utilizado para a plotagem do gráfico, temos que as coordenadas destes pontos são: $A(2,0358 ; 108,1647), B(3,1416 ; 107,4089)$ e $C(4,2473 ; 108,1647)$.

As abcissas que maximizam $\Psi(\alpha=2,0358 \mathrm{rad}$ ou $\alpha=4,2473 \mathrm{rad})$ também maximizam $S$, assim, o máximo valor da soma das capacidades dos dois filtros cônicos é:

$S(2,0358)=S(4,2473) \cong 0,45664 G^{3}$.

Temos que o ponto $B$ está associado ao padrão de corte diametral, de forma que os dois filtros tenham o mesmo formato. Ou seja, sejam idênticos, com capacidade $V(\pi)=\frac{\sqrt{3} \pi}{24} G^{3} \cong$ $0,2267 G^{3}$. Portando neste caso, a soma da capacidade dos dois filtros é aproximadamente $0,4534 G^{3}$.

O padrão ótimo de corte neste caso sem descarte é de corte em dois setores, sendo um deles de $2,0358 \mathrm{rad}\left(116,6^{\circ}\right)$ e o outro $4,2473 \mathrm{rad}\left(243,4^{\circ}\right)$.

Comparando o primeiro caso com descarte, e este sem descarte, verificamos que no primeiro o corte é feito de modo a maximizar apenas um dos filtros, onde o setor utilizado possui ângulo $\alpha \cong 5,1302$ radianos, e o setor descartado possuía ângulo $(2 \alpha-\pi) \cong 1,1530$ radianos. Calculando a soma dos volumes das capacidades dos 2 filtros, caso fosse construído um filtro com o setor descartado, teríamos $S(5,1302)=S(1,1530) \cong 0,43773 G^{3}$, ou seja, um valor menor que a soma ótima encontrada.

\section{DISCUSSÃO}

Através da resolução de exercícios e seminários apresentados durante o desenvolvimento do trabalho foi possível verificar os diversos ramos em que se é possível trabalhar com a matemática aplicada, principalmente quando relacionado com industrias, pois lá são trabalhadas diversas grandezas, e as vezes se é requerido a otimização de certa variável, como por exemplo filtros, onde se tem como objetivo a otimização dele para obter sua capacidade máxima.

\section{CONCLUSÃO}

Através do presente trabalho foi possível verificar a importância da matemática para a otimização de variáveis em diversos ramos industriais, como por exemplo, a otimização de um filtro cônico, de maneira a se obter sua capacidade máxima, minimizando assim a perda de matéria prima, e melhor utilização da matéria prima. 


\section{REFERÊNCIAS}

Lima, E. L. ; Morgado, A. C. O. ; Wagner, E. A Matemática do Ensino Médio. Coleção Do Professor de Matemática, Vol 3. Rio, Editora da SBM, 2003.

Costa, S. I. R. Otimizando Formas e Trajetórias. Campinas, IMECC/Unicamp, 2002.

Tunala, N. Projetando Filtros Cônicos de Capacidade Máxima. Revista do Professor de Matemática (RPM), No. 92, 2016. 